\title{
Cyclic Voltammetric Studies on the Role of Electrode, Electrode Surface Modification and Electrolyte Solution of an Electrochemical Cell
}

\author{
S.P. Mundinamani, M.K. Rabinal* \\ Department of Physics, Karnatak University Dharwad, India-580003. \\ Correspondence Author: mkrabinal@yahoo.com
}

\begin{abstract}
The present study has been aimed to understand the role of electrode material, its surface modification by organic molecules, solvent and analyte in an electrochemical cell on cyclic voltammograms. To study these effects, the different electrochemical cells were constituted using different electrode material, different solvents and thiol modification of electrode surface to measure the cyclic voltammograms. The present measurements clearly show that these parameters are significantly important ones in dictating the nature of the observed voltammograms. The effect of scan rate on cyclic voltammograms has been observed with various scan rates. A plot of the Randles-Sevcik equation yield a straight line, the slope is used to determine the diffusion coefficient. Since the cyclic voltammetry is an important technique in the characterization and in the development of one of the important energy storage devices that are known as supercapacitors. The present investigations clearly help to understand the internal mechanisms of energy storage in these devices so that the better devices can be developed.
\end{abstract}

Keywords: Cyclic Voltammograms, $\mathrm{SnO}_{2}: \mathrm{Sb}$, Carbon, Specific Capacitance, Randles-Sevcik relation.

\section{Introduction}

The cyclic voltammetry, a three electrode electrochemical cell, is an important technique in the analyses of electrochemical reactions between ions and surface atoms of electrodes under the investigation [1]. The method involves a linear and a cyclic variation of electrode potential between the working and reference electrodes within a potential window by measuring the current that flows between working and counter electrodes [2]. The technique provides an qualitative information on electrode reaction mechanisms, several quantitative properties of the charge transfer reactions between electrolyte ions and electrons with respect to the electrode surface can also be obtained [2,3]. The potential of these electrodes is a controlled parameter that causes the chemical species to be oxidized or reduced by removal or by addition of electrons to the ions by the electrode. The potential can be thought of as electron pressure which either forces a species in solution to gain an electron (reduction) or lose an electron (oxidation) [4]. As the potential of the electrode becomes more negative, it becomes more strongly reducing. Conversely, as the potential becomes more positive, it becomes more strongly oxidizing. Therefore, the reduction/oxidation (redox) reaction taking place on the electrode can be controlled by controlling the electrode potential of an electrochemical cell [5]. Hence, the cyclic voltammetry is a powerful tool to study the electrochemical behavior of a system by systematic study of current-voltage measurements of a given electrochemical cell.

The electrochemical capacitors that are also referred as "supercapacitors" or "ultracapacitors" are gaining a wide importance in modern science and technology due to their superior charge storage/charge delivery response in comparison with the other energy storage devices [6]. The specific parameters that help to improve the performance of these devices are the good electrical conductivity, large surface area, specific porosity, surface polarizability and the stability of electrode materials and high decomposition voltage, high dielectric conductivity, better stability, fast ionic diffusion, low cost and environmental compatibility are the essential properties of electrolytes [7]. In addition to the large surface area to enhance the specific capacitance of these devices, recently it has been suggested that the inclusion of a suitable analyte/redox couple in the electrolytic solution and chemical bonding of certain organic/bio-molecules on electrode surface also help in the significant improvement in the specific capacitance $[8,9]$.

It has been well established that the cyclic voltammetry is the most suitable technique to study all such mechanisms in a greater detail [10]. But the certain issues like the condition of electrode surface, surface modification, role of solvent, presence of redox couple and the kinetic dependence of redox peaks on scan rate etc are need to be understand more properly. The present investigations are aimed to study the detailed cyclic voltammetry of electrochemical cell by changing the certain parameters like the different electrode materials, different solvents (polar), and the role of background electrolyte, analyte or reactant / redox couple, also the chemical modification of electrode surface by organic molecules. 


\section{Experimental Section}

All the chemicals and the reagents are used of analytical grade, these are used as they received without further purification. Commercially available Camlin $0.7 \mathrm{~mm}$ graphite rods $(0.07 \mathrm{~cm}$ diameter and $6 \mathrm{~cm}$ of length) are used as carbon electrode material to study the cyclic voltammetry. A highly conducting thin films of tin oxide doped with antimony $\left(\mathrm{SnO}_{2}: \mathrm{Sb} 4\right.$ wt.\%) on glass surface are also used as electrode materials. The details of synthesis and characterization of these films with antimony composition are discussed elsewhere [11]. Ethylene glycol $\left(\mathrm{C}_{2} \mathrm{H}_{6} \mathrm{O}_{2}\right)$, potassium nitrate $\left(\mathrm{KNO}_{3}\right)$ and potassium ferricyanide $\left(\mathrm{K}_{3} \mathrm{Fe}[\mathrm{CN}]_{6}\right)$ are obtained from the Hi-Media chemicals, India. Heptanethiol $\left(\mathrm{C}_{7} \mathrm{H}_{16} \mathrm{~S}\right)$ is procured form Sigma-Aldrich chemicals. This molecule is used for the surface modification of carbon electrode. For all the washing and reaction purpose tripled distilled water (Milli-Q water) is used.

For the construction of electrochemical cell, $1 \mathrm{M}$ of $\mathrm{KNO}_{3}$ solution is prepared as an electrolyte and 10 $\mathrm{mM}$ solution of potassium ferricyanide $\left(\mathrm{K}_{3} \mathrm{Fe}[\mathrm{CN}]_{6}\right)$ is prepared in water as a redox couple. To make a standard solution of this redox couple, in $9.5 \mathrm{ml}$ of $\mathrm{KNO}_{3}$ solution $0.5 \mathrm{ml}\left(\mathrm{K}_{3} \mathrm{Fe}[\mathrm{CN}]_{6}\right)$ solution was added. Similar standard is prepared in ethylene glycol in the place of water. To form electrochemical cell the two electrode configuration cell (Swagelok type configuration) is used, in which working electrode acts as one of the electrode and shorting of counter electrode with reference electrode acts as another. This two electrodes cell is used to study the above mentioned aspects using different electrodes and solvents. Further, the electrochemical grafting of organic molecule (Heptanethiol $\left(\mathrm{C}_{7} \mathrm{H}_{16} \mathrm{~S}\right)$ ) on the carbon surface is carried by passing constant current of 100 $\mu \mathrm{A}$ for 2 hours duration in a $50 \mathrm{mM}$ organic molecules solutions and the electrode well rinsed in absolute ethanol to remove the loosely bounded molecules. The measurement of cyclic voltammograms were carried out using the ACM instruments Gill AC potentiostat device.

\section{Results And Discussion}

In the present work, to understand the effects of solvent, electrodes and molecular modification of electrodes on cyclic voltammograms with respect to $\mathrm{K}_{3} \mathrm{Fe}[\mathrm{CN}]_{6}$ is carried out using the two electrode (Swagelok) configuration as an electrochemical cell. As mentioned above, here the reference and counter electrodes of equipment are shorted as one electrode and working electrode as another electrode. The separation between the two electrodes are mentioned almost constant $(1 \mathrm{~cm})$ in all the measurements that are reported here. The scientific reliability and the simplicity of two electrode configuration over the conventional three electrode configuration of a electrochemical cell in the study of electrochemical capacitors has been well documented by Stoller et al. [12] and others [13]. The best practices on the use of this setup are well summarized by the Stoller et al. [12]. Further, recently we also well testified the reliability of this setup in the characterization of supercapacitors [14]. Firstly, two symmetrical carbon electrodes are immersed in electrolyte solution (in this case the distilled water and ethylene glycol are used as an electrolyte). The CV curves that are recorded under the scan rate of $120 \mathrm{mV} / \mathrm{s}$. Figure 1(a) shows the measured CV curves under the potential window ranging from -1 to $+1 \mathrm{~V}$. It can be clearly seen that the two scans are quite similar in nature but they differ in their current levels. For water, the current level is high and the CV curve is rectangular in shape, the later indicates that the capacitor behaves as purely electrostatic one. In case of ethylene glycol the current level is less and the curve slightly deviates from a pure electrostatic behavior. These measurements clearly suggest that the solvents do play a role in controlling the nature and current levels in the cyclic voltammograms and hence in the performance of electrolytic capacitors. Next, these measurements were repeated with respect to the different electrodes that are highly conducting $\mathrm{SnO}_{2}: \mathrm{Sb}$ films deposited on glass surface. These plots are shown in figure 1(b). It is evident from these graphs when compared with the graphs of figure 1(a) that there is clear enhancement in the current levels with respect to $\mathrm{SnO}_{2}: \mathrm{Sb}$ as compared to carbon electrodes for similar electrolytes. The change of electrodes from carbon to $\mathrm{SnO}_{2}: \mathrm{Sb}$ has enhanced the area under the curves that is directly proportional to the specific capacitance of an electrolytic capacitor [6,15-17]. 


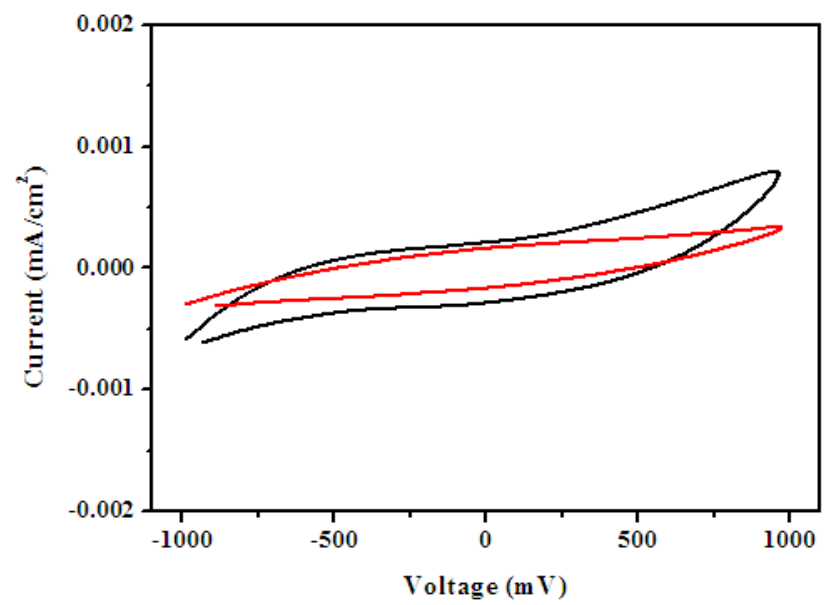

Figure 1(a): Cyclic voltammograms of carbon electrodes in water (black line) and ethylene glycol solvents (red line) as an electrolyte species.

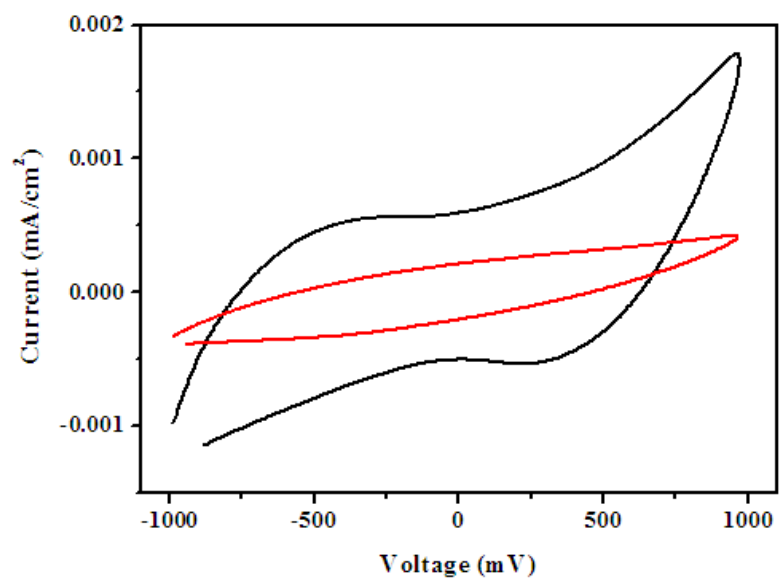

Figure 1(b): Cyclic voltammograms of $\mathrm{SnO}_{2}$ :Sb electrodes in water (black line)and ethylene glycol (red line) solvents.

The above cyclic voltammograms were repeated by introducing $\mathrm{KNO}_{3}$ to understand the role of electrolyte addition on the cyclic voltammograms. These curves are shown in figures 2(a) and 2(b) for symmetric carbon and $\mathrm{SnO}_{2}$ : $\mathrm{Sb}$ electrodes. In case of carbon electrodes (figure 2(a)), there is an almost three fold increase in the current levels. A similar change is also observed in case $\mathrm{SnO}_{2}: \mathrm{Sb}$ electrodes. However in the latter case broad humps are clearly observed that leads to greater area under the CV curves.

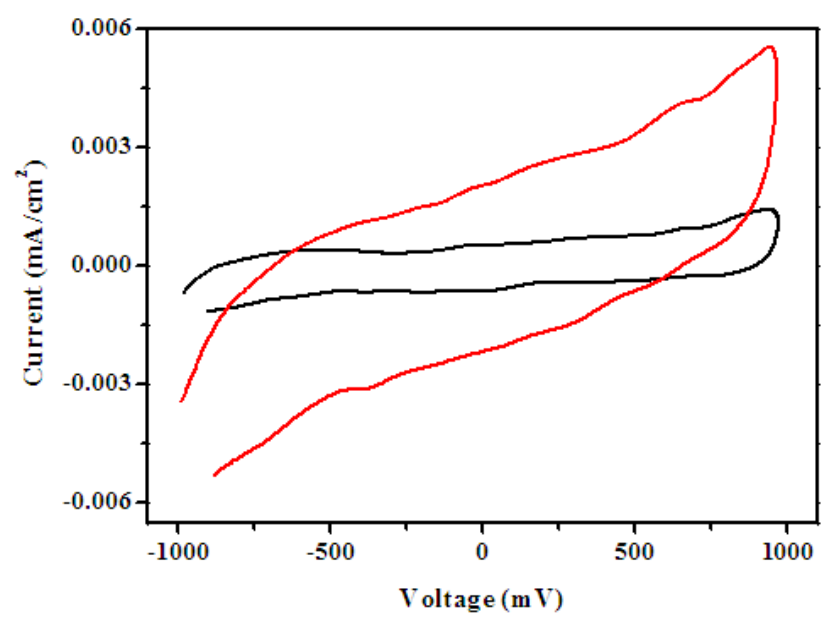

Figure 2(a): Cyclic voltammograms of carbon electrodes in water with $\mathrm{KNO}_{3}$ (black line)and ethylene glycol with $\mathrm{KNO}_{3}$ (red line) mixture as an electrolyte species 


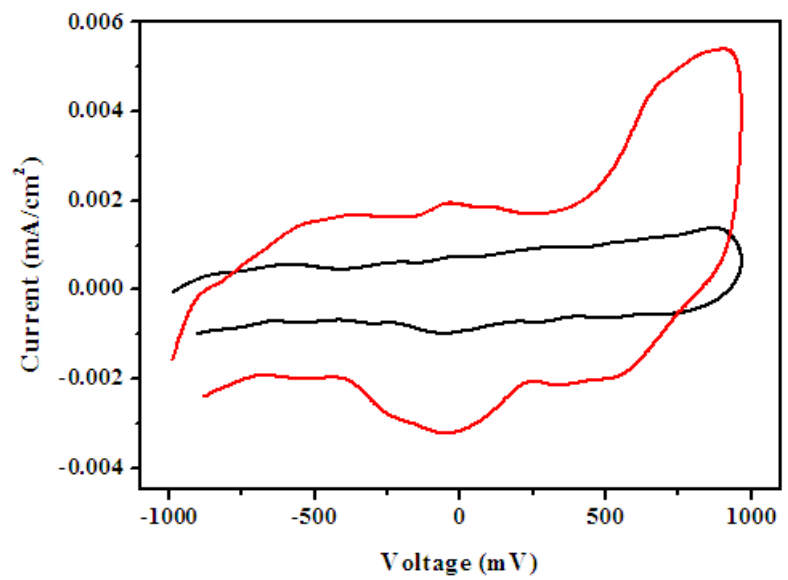

Figure 2(b): Cyclic voltammograms of $\mathrm{SnO}_{2}: \mathrm{Sb}$ electrodes in water with $\mathrm{KNO}_{3}$ (black line) and ethylene glycol with $\mathrm{KNO}_{3}$ (red line) mixture as an electrolyte species.

The curves in figure 2(b) strongly deviate from the rectangular nature indicating that the mechanisms other than purely electrostatic also play a rule. The increase in current levels in these plots as compared to plots of figure 1 are due to ion diffusion, $\mathrm{KNO}_{3}$ in water and $\mathrm{EG}$ produces the easy access of $\mathrm{K}^{+}$ions reaching the electrode surface.

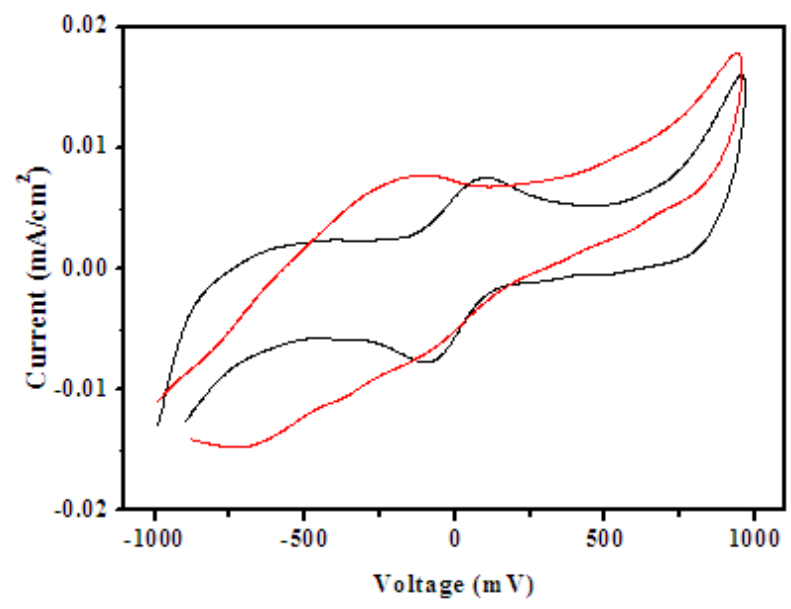

Figure 3(a): Cyclic voltammograms of carbon electrodes in water with $\mathrm{KNO}_{3}$, potassium ferricyanide (blackline) and ethylene glycol with $\mathrm{KNO}_{3}$, potassium ferricyanide(red line).

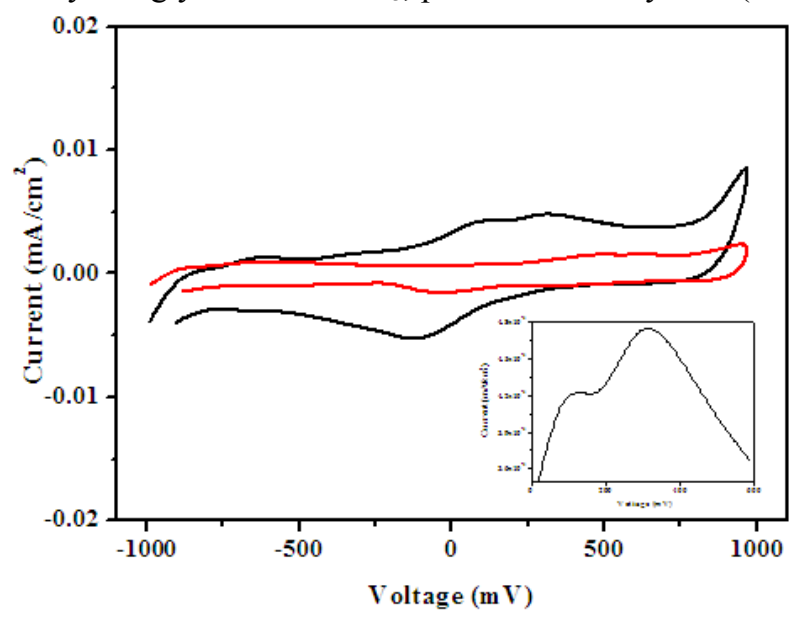

Figure 3(b): Cyclic voltammograms of $\mathrm{SnO}_{2}: \mathrm{Sb}$ electrodes in water with $\mathrm{KNO}_{3}$, potassium ferricyanide (black line) and ethylene glycol with $\mathrm{KNO}_{3}$, potassium ferricyanide (red line). The inset in the figure is enlarged view of the plot between the voltage $0 \mathrm{mV}$ and $600 \mathrm{mV}$ to make the appearance of two peaks more clear. 
Further, the above measurements were repeated with carbon and $\mathrm{SnO}_{2}: \mathrm{Sb}$ symmetric electrodes by introducing an redox couple that is $\mathrm{K}_{3} \mathrm{Fe}[\mathrm{CN}]_{6}$. Now the electrolyte between the carbon-carbon and $\mathrm{SnO} 2: \mathrm{Sb}-$ $\mathrm{SnO}_{2}: \mathrm{Sb}$ electrodes consist of water/ethylene glycol- $\mathrm{KNO}_{3}-\mathrm{K}_{3} \mathrm{Fe}[\mathrm{CN}]_{6}$ in order to study the cyclic voltammograms. In case of carbon-carbon electrodes the curves with respect to water and ethylene glycol are shown in figure 3(a). Interestingly in the case of water, the reduction and oxidation peaks are clearly visible in these curves, the oxidation peak occurs at $103 \mathrm{mV}$ and the reduction peak at $-81 \mathrm{mV}$. In case of standard $\mathrm{K}_{3} \mathrm{Fe}(\mathrm{CN})_{6}$ electrolyte oxidation peak occurs at $300 \mathrm{mV}$ and reduction peak at $200 \mathrm{mV}$ with respect to three electrode configuration in which glassy carbon as working electrode, $\mathrm{Ag} / \mathrm{AgCl}$ electrode as reference electrode and platinum as counter electrode are used. In comparison with this, the standard redox peaks in the present case are shifted by close to $0.2 \mathrm{~V}$. This could be attributed to the electrode and electrolyte interactions. Whereas for ethylene glycol as a solvent these symmetric oxidation and reduction peaks are completely absent. But there is a broad hump as an oxidation peak at $250 \mathrm{mV}$ while scanning from the lower potential to the higher potential. In both water and ethylene glycol the additives were completely dissolved and are of equal molarity. The sluggish behavior of ethylene glycol could be due to its high viscosity that prevents the fast diffusion of ions from electrolyte to the electrode surface. The broad hump/peak (in the oxidation side) is due to the release of an electron from the surface of electrode. The ethylene glycol is preventing the electron to reach the $\mathrm{Fe}^{2+}$ destination. In case of water as a solvent $\mathrm{Fe}^{3+}$ after losing an electron becomes $\mathrm{Fe}^{2+}$ (oxidation) while $\mathrm{Fe}^{2+}$ after gaining one electron becomes $\mathrm{Fe}^{3+}$ (reduction). In case of $\mathrm{SnO}_{2}: \mathrm{Sb}_{-} \mathrm{SnO}_{2}: \mathrm{Sb}$ electrodes the results are plotted in figure 3(b). For water, the redox peaks are clearly seen with two oxidation peaks one at $90 \mathrm{mV}$ and another at $320 \mathrm{mV}$. The expanded curves of this region is also shown as an inset in figure 3(b) for the clarity, the two broad peaks are clearly visible. Here the current levels are low as compared to carbon electrodes. The reduction peak occurs at $-105 \mathrm{mV}$.

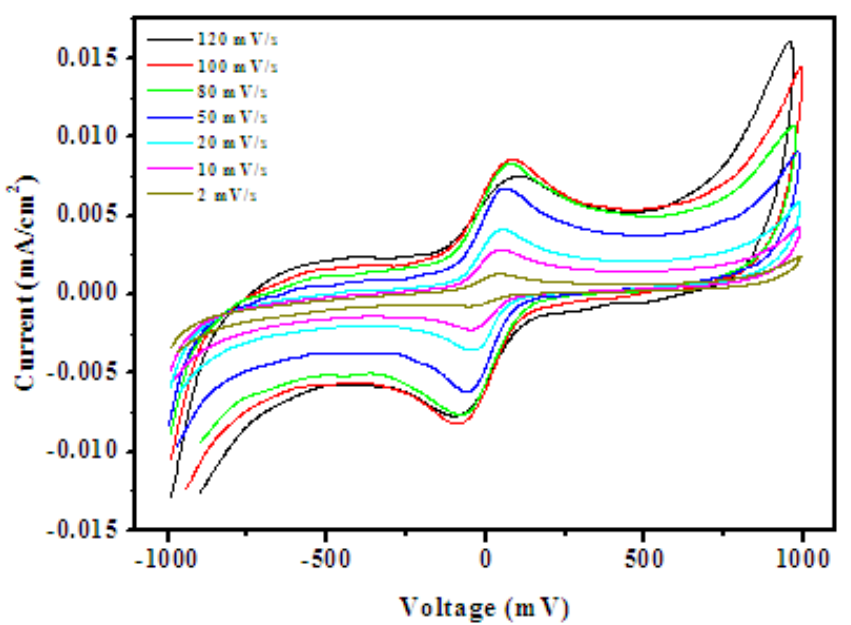

Figure 4(a): Cyclic voltammograms with different scan rates of carbon electrodes, in water along with $\mathrm{KNO}_{3}$, potassium ferricyanide mixture as an electrolyte.

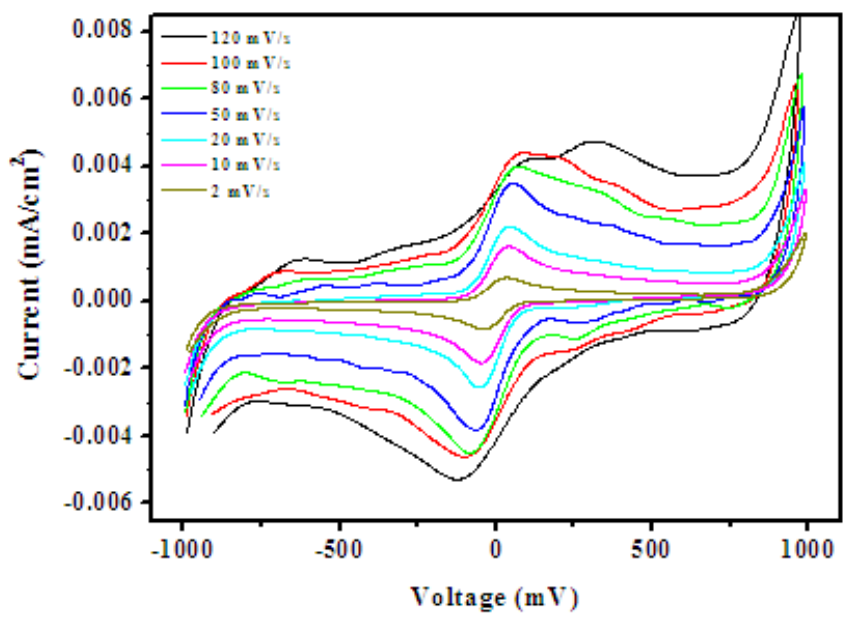

Figure 4(b): Cyclic voltammograms with different scan rates of $\mathrm{SnO}_{2}$ : $\mathrm{Sb}$ electrodes, in water along with $\mathrm{KNO}_{3}$, potassium ferricyanide mixture as an electrolyte. 


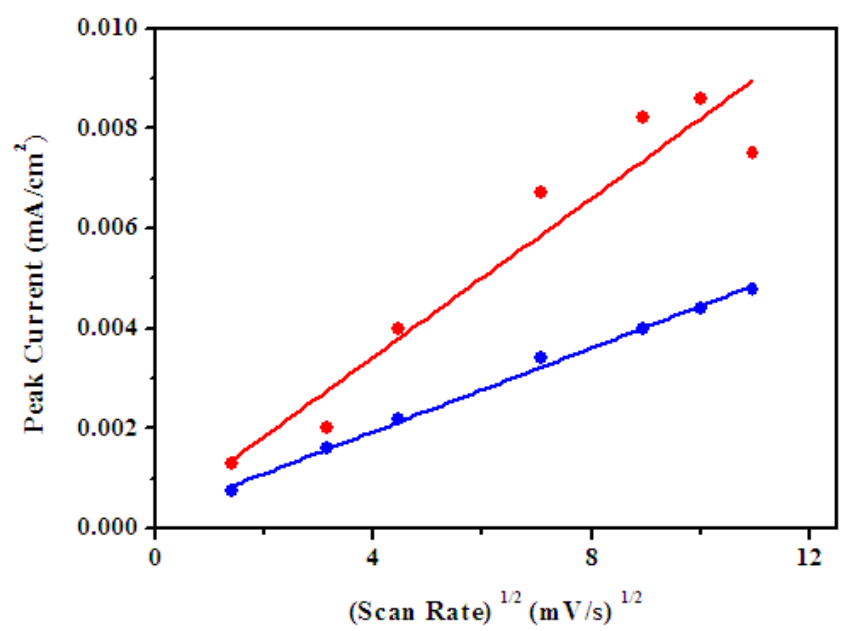

Figure 5: For carbon electrodes cell the Randles-Sevcik relation is plotted, peak currents for different scan rates versus the square root of scan rates (red line). It is also plotted in case of $\mathrm{SnO}_{2}$ : $\mathrm{Sb}$ electrodes (blue line).

It has been a well established fact that the reduction and oxidation process in an electrochemical reaction are kinetic in nature. There is a systematic shift in the redox peak position with voltage scan rate in cyclic voltammetric measurements [18,19]. A theoretical model has been developed by Randles and Sevcik to predict how the peak current in reduction /oxidation process dependents on the voltage scan rate [20]. The relation between these parameters is given by the following formula that is known as Randles-Sevcik equation

$$
i_{p}=0.4463 n \text { FAC }\left(\frac{n F v D}{R T}\right)^{\frac{1}{2}}
$$

where $i_{p}$ is the peak current of reduction/oxidation process, $n$ is the number of electrons per reaction species (for $\mathrm{Fe}^{2+}$ it is 1), $\mathrm{A}$ is the area of electrode in contact with electrolytic solution, $\mathrm{D}$ is the diffusion coefficient, $\mathrm{C}$ is the electrolyte concentration, $\mathrm{F}$ is faraday constant, $\mathrm{R}$ is Rydberg constant, $\mathrm{T}$ is the temperature and $\mathrm{v}$ is the scan rate. This equation clearly predicts that the reduction/oxidation peak position is proportional to the square root of scan rates. In the present measurements, the Randles-Sevcik relation has been studied for carbon-carbon and $\mathrm{SnO}_{2}: \mathrm{Sb}_{-}-\mathrm{SnO}_{2}: \mathrm{Sb}$ electrodes in the presence of water- $\mathrm{KNO}_{3}-\mathrm{K}_{3} \mathrm{Fe}[\mathrm{CN}]_{6}$ electrolyte, since this electrolyte clearly exhibits the reduction/oxidation peaks. The scanning is done in the range from $2,10,20$, $50,80,100$ to $120 \mathrm{mV} / \mathrm{s}$. These curves in case of carbon-carbon electrodes are shown in figure 4(a) and that for $\mathrm{SnO}_{2}: \mathrm{Sb}_{-} \mathrm{SnO}_{2}: \mathrm{Sb}$ electrodes are shown in figure 4(b). A clear shift in reduction peaks with scan rate is evident from these plots. In case of former there is single reduction peak in all the scans and in later case only at smaller scan rates this is a single peak but it splits into two peaks from $80 \mathrm{mV} / \mathrm{s}$ onward and the two peaks are very prominent at $120 \mathrm{mV} / \mathrm{s}$. The Randles-Sevcik equation is used to plot the peak current values as function of square root of scan rate, these plots for carbon-carbon and $\mathrm{SnO}_{2}: \mathrm{Sb}_{-} \mathrm{SnO}_{2}: \mathrm{Sb}$ electrodes are shown in figure 5, respectively. In both the cases there is almost linear relation between these parameters indicating that in both the cells, the equation (1) is obeyed. However, the fit is better in later case as compared to the former. Further, from these plots using equation (1) the ionic diffusion coefficients are estimated. These are $8.101 \times 10^{-3}$ and $3.945 \mathrm{x}$ $10^{-3} \mathrm{~cm}^{2} \mathrm{~s}^{-1}$ in case of carbon-carbon and $\mathrm{SnO}_{2}: \mathrm{Sb}_{-} \mathrm{SnO}_{2}: \mathrm{Sb}$ electrodes, respectively. Though the electrolyte is same in both the electrochemical cells, this clearly indicates that the electrode plays an important role in controlling the ionic diffusion. 


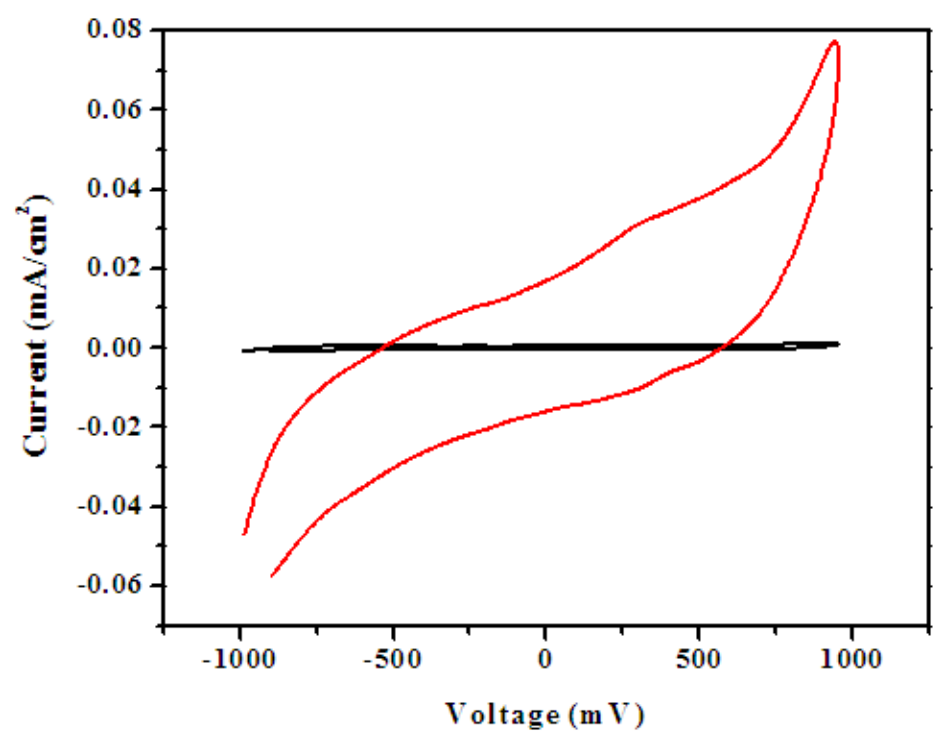

Figure 6(a): Cyclic voltammograms of bare carbon electrodes (black line) and molecularly modified carbon electrodes (red line) in water with $\mathrm{KNO}_{3}$ mixture as an electrolyte that is measured under 120 $\mathrm{mV} / \mathrm{s}$ scan rate.

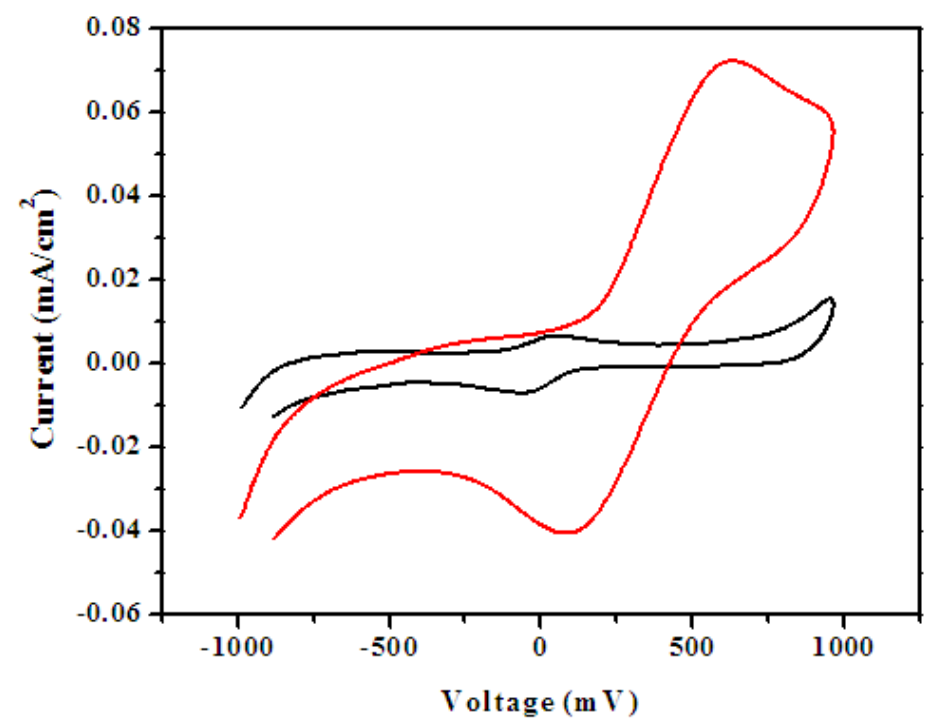

Figure 6(b): Cyclic voltammograms of bare carbon electrodes (black line) and molecularly modified carbon electrodes (red line) in water with $\mathrm{KNO}_{3}$, potassium ferricyanide mixture as an electrolyte and the scan is recorded with $120 \mathrm{mV} / \mathrm{s}$.

As mentioned above, the chemical binding of certain molecules and biomolecules significantly enhances the current level and generates new peaks in the cyclic voltammograms [8,9]. In order to study this effect in case of carbon electrode, heptanethiol molecules are chosen to bind on the surface of electrode by electrografting process. First, the chemically modified carbon-carbon electrodes are used in water- $\mathrm{KNO}_{3}$ electrolyte. These measurements are plotted in figure 6(a), for comparison the curve of bare (chemically unmodified) electrodes is also shown. The significant difference between these curves is that there is large enhancement in the current levels at given potential. For bare electrode it is observed that $0.001 \mathrm{~mA} / \mathrm{cm}^{2}$ is the current order at $1 \mathrm{~V}$, while for the molecularly modified electrodes show the current order of $0.07 \mathrm{~mA} / \mathrm{cm}^{2}$ at 1 $\mathrm{V}$ that is almost one order of magnitude more. It clearly shows that the molecular binding on electrode surface has a major role in controlling the current in cyclic voltammograms. Further, these measurements are repeated in the presence of redox couple $\mathrm{K}_{3} \mathrm{Fe}[\mathrm{CN}]_{6}$. These curves are shown in figure 6(b) for both the bare and the chemically modified carbon electrodes. Once again the chemically modified electrode surface enhances the current level by more than one order. The redox peaks are significantly shifted to higher voltage as compared to bare electrodes. In case of chemical modification the reduction occurs at $600 \mathrm{mV}$ whereas the oxidation occurs 
at $100 \mathrm{mV}$. All these results suggest that the ion diffusion in vicinity of electrode-electrolyte interface gets enhanced after chemical modification. This clearly demonstrates that the molecular modification of electrode surface in an electrochemical cell is an important aspect to enhance the specific capacitance.

\section{Conclusions}

In the present work a systematic investigations are carried out to study the role of electrodes, solvents and the chemical modification of electrode surfaces on the nature of current-voltage curves of cyclic voltammetry. These measurements clearly suggest that the nature and level of currents in cyclic voltammograms clearly depend on the many factors. These include the nature of electrode material, the type of solvents and scan rates. The expected reduction/oxidation peak intensity and position a redox couple also dependent on solvent and electrode material. Further, the chemical binding of certain molecules on electrode surface is very important in the enhancement of current levels and shift in the reduction/oxidation peak position. This gives raise to high area under cyclic voltammogram and hence a high value of specific capacitance of a supercapacitor. This strategy would be an interesting aspect in the development of supercapacitors for the modern applications.

\section{Acknowledgements}

One of the authors SPM would like to thank UGC, India, for helping the financial support in the RFSMS scheme.

\section{References}

[1]. Princeton Applied Research, Applied Instrument Group, Basics of voltammetry and polarography, Application Note P-2, 1-12.

[2]. J. Wang, Analytical Electrochemistry, Chapter 2, John Wiley \& Sons (2000).

[3]. R.S. Nicholson and I. Shain, Theory of stationary electrode polarography, single scan and cyclic methods applied to reversible, irreversible and kinetic systems, Anal. Chem, 36, 1964, 706-723.

[4]. D.K. Gosser, Jr., Cycic Voltammetry: Simulation and Analysis of reaction mechanisms, VCH Publishers (1993).

[5]. A.J. Bard and L.R. Faulkner, Electrochemical Methods: Fundamental and Applications, Second Edition, John Wiley and Sons Publishers (2001).

[6]. P. Simon and Y. Gogotsi, Materials for electrochemical capacitors, Nature Materials, 7, 2008, 845-855.

[7]. G. Wang, L. Zhang and J. Zhang, A review of electrode materials for electrochemical supercapacitors, Chem. Soc. Rev, 41, 2012, 797-828.

[8]. Q. Wu, Y. Sun, H. Bai and G. Shi, High-performance supercapacitor electrodes based on graphene hydrogels modified with 2aminoanthraquinone moieties, Phy. Chem. Chem. Phys, 13, 2011, 11193-11198.

[9]. M. Khairy and S.A. El Safty, Hemoproteins-nickel foam hybrids as effective supercapacitors, Chem. Comm, 50, 2014, $1356-1358$.

[10]. B.E. Conway, Electrochemical Supercapacitors: Scientific Fundamentals and Technological Applications, (Kluwer, 1999).

[11]. Uma Nerle, R.M. Hodlur and M.K. Rabinal, A sharp and visible range plasmonic in heavily doped metal oxide films, Materials Research Express, 1, 2014, 015910, 1-17.

[12]. M.D. Stoller and R.S. Rouff, Best practice methods for determining an electrode materials performance for ultracapacitors, Energy and Environmental Science, 3, 2010, 1294-1301.

[13]. S. Ratha and C.S. Rout, Supercapacitor Electrodes Based on Layered Tungsten Disulfide-Reduced Graphene Oxide Hybrids Synthesized by a Facile Hydrothermal Method, ACS Appl. Mater. Interfaces, 5, 2013, 11427-11433.

[14]. S.P. Mundinamani and M.K. Rabinal, Molecular modification of highly degenerate semiconductor as an active electrode to enhance the performance of supercapacitor, J.Physics.D: Appl Phy, 2014 Communicated.

[15]. P. Simon and A. Burkey, Nanostructured carbons: Double layer capacitance and more, The electrochemical Interface, Spring 2008, $38-43$.

[16]. C. Zhao, W. Zheng, X. Wang, H. Zhang, X. Cui and H. Wang, Ultrahigh capacitive performance from both Co(OH) $)_{2} /$ graphene electrode and $\mathrm{K}_{3} \mathrm{Fe}(\mathrm{CN})_{6}$ electrolyte, Scientific Reports, 3:2986, 2013, 1-6.

[17]. W. Chen, Z. Fan, L. Gu, X. Bao and C. Wang., Enhanced capacitance of manganese oxide via confinement inside carbon nanotubes, Chem. Comm, 46, 2010, 3905-3907.

[18]. X. Wang, Q. Xiang, B. Liu, L. Wang, T. Luo, D. Chen and G. Shen, TiO 2 modified FeS nanostructures with enhanced electrochemical performance for lithium-ion batteries, Scientific Reports, 3:2007, 2013, 1-8.

[19]. B. Zhang, Y. Yu, Z. Huang, Y-B. He, D. Jang, W-S. Yoon, Y-W. Mai, F. Kang and J-K. Kim, Exceptional electrochemical performance of free standing electrospun carbon nanofiber anodes containing ultrafine $\mathrm{SnO}_{\mathrm{x}}$ particles, Energy Environ. Sci, 5, 2012, 9895-9902.

[20]. S.R. Das, S.B. Majumder and R.S. Katiyar, Kinetic analysis of the $\mathrm{Li}^{+}$ion intercalation behaviour of solution derived nanocrystalline lithium manganate thin films, J. Power Source,139, 2005, 261-268. 